\section{Amyloid Myopathy as an Inclusion Body Myositis Mimic}

Anai C. Hamasaki, MD ${ }^{1}$, Ryan D. Jacobson, MD², Barbara J. Distad, MD ${ }^{6}$, Michael D. Weiss, MD ${ }^{6}$, Jeffrey M. Statland, MD ${ }^{2}$, Omar Jawdat, MD ${ }^{2}$, Melanie D. Glenn, $\mathrm{MD}^{2}$, Laura Herbelin, $\mathrm{BS}^{2}$, Richard J. Barohn, $\mathrm{MD}^{2}$, Mamatha Pasnoor, $\mathrm{MD}^{2}$, Gary W. Gallagher, $\mathrm{MD}^{4}$, Sandra I. Camelo-Piragua, $\mathrm{MD}^{5}$, Andrew J Heim, CCRP2, Mazen M. Dimachkie, $\mathrm{MD}^{2}$

${ }^{1}$ Department of Neurology, The University of Oklahoma Health Sciences Center, Oklahoma City, OK

${ }^{2}$ Department of Neurology, The University of Kansas Medical Center, Kansas City, KS

${ }^{3}$ Department of Neurology, Loyola University Chicago, Chicago, IL

${ }^{4}$ Department of Neurology, University of Michigan, Ann Arbor, MI

${ }^{5}$ Department of Pathology, University of Michigan, Ann Arbor, MI

${ }^{6}$ Department of Neurology, University of Washington, Seattle, WA

\section{ABSTRACT}

Introduction: Amyloid myopathy is a rare presentation of systemic amyloidosis. Amyloid myopathy can be initially misdiagnosed as sporadic inclusion body myositis (IBM).

Methods: We report 4 cases of amyloid myopathy clinically mimicking inclusion body myositis and initially thought to be phenotypically IBM by neuromuscular experts.

Results: Case 1 is an 81-year-old woman who presented with distal arm and proximal leg asymmetric weakness (myopathy pattern 4). Case 2 is a 76-year-old man with primary systemic amyloidosis who presented with myopathy pattern 4 and progressive dysphagia for four years. Case 3 is an 82-year-old man with progressive myopathy pattern 4 weakness and swallowing difficulty. Case 4 is a 62 -yearold man with progressive bilateral finger flexor weakness. Muscle biopsies in all 4 cases showed perivascular amyloid deposits.

Discussion: Amyloid myopathy may be clinically indistinguishable from IBM. Muscle biopsy is of critical importance in the evaluation of patients suspected to have IBM.

Keywords: amyloid, myopathy, amyloidosis, IBM, myositis.

\section{Introduction}

Primary systemic amyloidosis is a disease resulting from deposition of amyloid in tissues causing organ dysfunction. Kidneys, heart, peripheral nerves and liver are commonly affected organs. ${ }^{1}$ Amyloid deposits are formed from monoclonal serum proteins in plasma cell dyscrasia ${ }^{2}$ and may result from deposition of heavy chain, monoclonal light chains or its N-terminal fragment. ${ }^{3}$

Amyloid myopathy is a rare presentation of primary systemic amyloidosis. Typically, it presents with proximal muscle weakness and elevated creatine kinase level. ${ }^{4,5}$

Sporadic inclusion body myositis (IBM) is the most common idiopathic inflammatory myopathy (IIM) after age $50,{ }^{6}$ characterized by distal upper extremity and proximal lower extremity weakness, also known as myopathy pattern 4 (MP4).

A literature search yielded two case reports of amyloid myopathy patients initially presenting with an IBM phenotype. ${ }^{5,8}$ We present four more cases from four large academic centers.

\section{Methods}

We report four cases of amyloid myopathy clinically mimicking inclusion body myositis and initially thought to be phenotypically IBM by neuromuscular experts.

\section{Results}

Case 1: An 81-year-old female was evaluated for progressive generalized weakness and dysphagia for two years. There was no similar family history. On examination, she had tongue enlargement (Supplementary Fig. 1), MP4 with asymmetric weakness right and left respectively in knee extension $3 / 4$, hip flexion $2 / 5$, finger flexion $4 / 3$ and shoulder abduction $4 / 4^{+}$. There was decreased pinprick below ankles, and ankle reflexes were absent. She was initially suspected to have IBM. Laboratory testing showed cre-

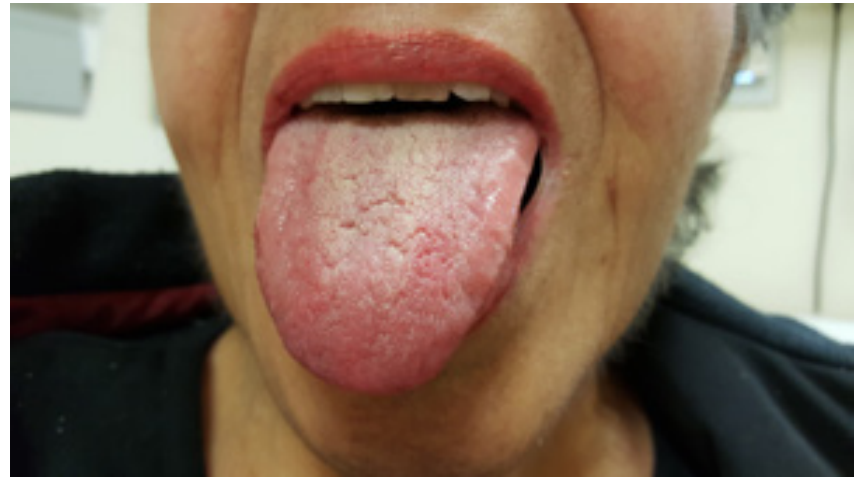

Supplementary Figure 1. Enlargement of the tongue of case 1. 
atine kinase (CK) of 141 IU/L, IgA monoclonal protein with lambda light chain in serum and urine and elevated serum beta-2 microglobulin. Electromyography (EMG) revealed an irritative myopathy. Biceps muscle biopsy demonstrated perivascular amyloid deposits indicative of amyloid myopathy (Figure 1). There was no evidence of heart involvement by transthoracic echocardiogram. She was treated with chemotherapy (cyclophosphamide, bortezomib and dexamethasone) along with physical therapy without response and ultimately required nursing home care. She developed postural hypotension due to associated autonomic neuropathy.
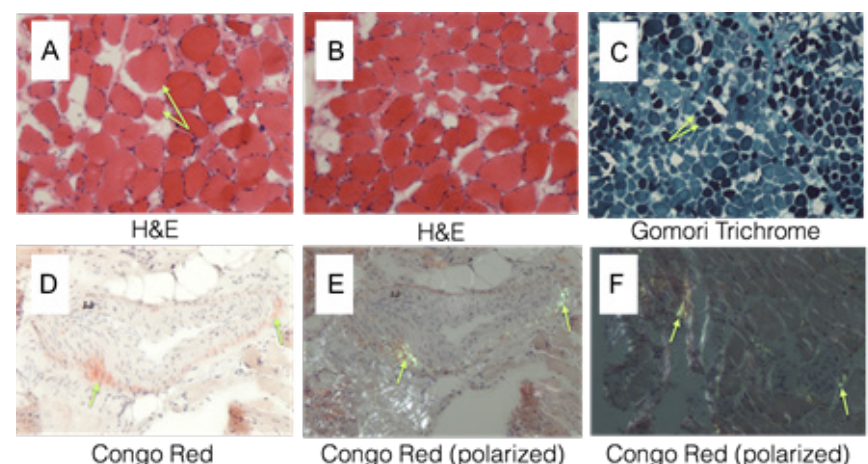

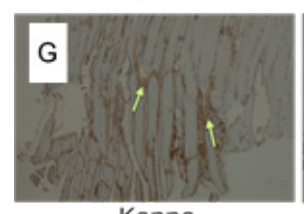

Kappa
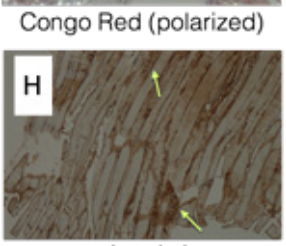

Lambda

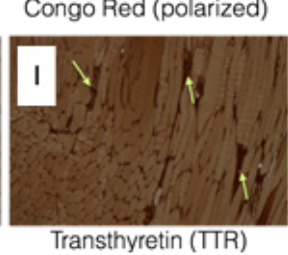

Figure 1. Biopsy of the biceps muscle. A) and B) Hematoxylin-Eosin (H\&E) and C) Gomori-Trichrome stains showing muscle fiber size variability. There is no inflammation or vacuolation. D) Congo red stain showing salmon-pink amyloid deposits in blood vessel wall. E) and F) Congo red stain under polarized light showing applegreen birefringent amyloid deposits in blood vessel and muscle fibers. G), H) and I) Immunohistochemical staining showing immunoreactivity against kappa light chain $(G)$, lambda light chain (H) and transthyretin (I).

Case 2: A 76-year-old male presented with progressive dysphagia for four years and thigh predominant leg weakness for four to five years. Around dysphagia onset, he was diagnosed with heart and renal failure due to primary amyloidosis. Despite chemotherapy, cardiac function declined. Neuromuscular examination demonstrated mild flaccid dysarthria and symmetric limb weakness. Leg weakness was mostly proximal, barely antigravity in quadriceps muscles. Arm weakness was most prominent distally with finger flexors involvement. There was no sensory loss or tongue enlargement. Initially, the patient was suspected to have IBM, despite known sys- temic amyloidosis diagnosis. EMG revealed myopathy with increased insertional activity. Vastus lateralis muscle biopsy revealed endomysial and perivascular amyloid deposits (Supplementary Fig. 2). There were no rimmed vacuoles nor inflammation. He died of presumed cardiac arrhythmia or aspiration one month following myopathy diagnosis.

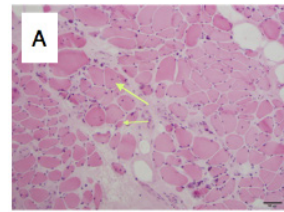

H\&E

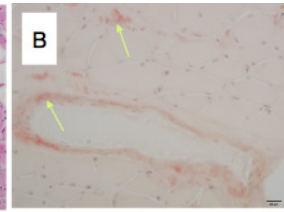

Congo Red

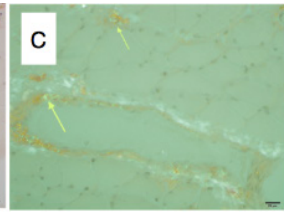

Congo Red (Polarized)
Supplementary Figure 2. Biopsy of the vastus lateralis muscle of case 3. A) H\&E stain showing muscle fiber size variability. B) Congo red stain showing salmon-pink amyloid deposits in blood vessel wall and muscle fibers. C) Congo red stain under polarized light showing apple-green birefringent amyloid deposits in blood vessel and muscle fibers.

Case 3: An 82-year-old male presented with 3 years of progressive proximal weakness. He had difficulty initially lifting arms above his head, followed by difficulty standing up and keeping his head up, then difficulty with buttoning and imbalance leading to falls. He also experienced swallowing difficulties and chronic constipation. Weakness was noted on neck flexion and extension, right greater than left proximal arms, and finger flexors greater than extensors, as well as antigravity strength in proximal legs and knee extensors. Reflexes were absent in the legs with reduced vibration. Lambda free light chain was elevated. EMG showed an irritative myopathy. Deltoid muscle biopsy showed myofiber necrosis, type II atrophy, mild fibrosis and upregulated HLA class 1 expression (Supplementary Fig. 3). Congo red stains revealed amyloid within arteriolar walls and endomysial connective tissues. Bone marrow biopsy showed presence of Lambda Bence Jones protein without evidence of amyloid deposition on Congo red stain.

Case 4: A 62-year-old male presented with a one and a halfyear history of progressive bilateral hand weakness, worse on the left. Examination showed weakness of flexor digitorum profundus $4+/ 3$ (digits 3,4 and 5) and 3+/2 (digits 1 and 2 ), right and left respectively. CK was $280 \mathrm{IU} / \mathrm{L}$. He had normal serum and urine electrophoresis, immunofixation electrophoresis, and kappa to lambda free light chain ratio. EMG showed irritative myopathy. He was initially thought 


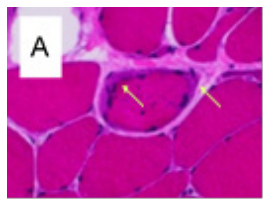

$H \& E$

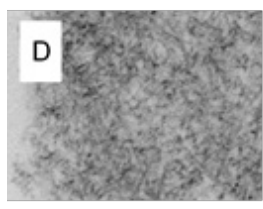

EM

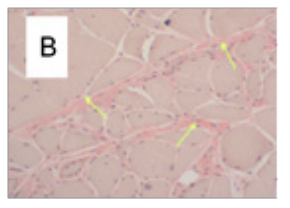

Congo Red

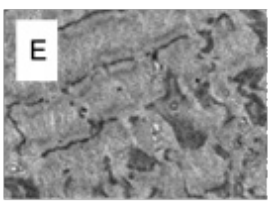

EM

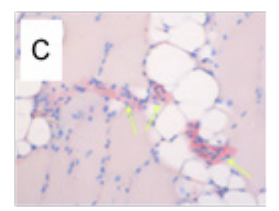

Congo Red

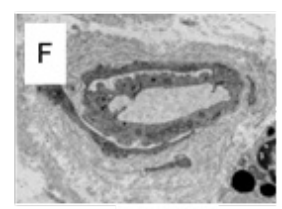

EM
Supplementary Figure 3. Biopsy of the right deltoid muscle. A) H\&E stain showing muscle fiber size variability and subsarcolemmal aggregates. B) Congo red stain showing muscle fiber size variability and interstitial thickening/ amyloid. C) Congo red stain showing vascular (right) and interstitial (center) amyloid. D) Electron microscopy (EM) 30,000x with interstitial amyloid fibrils, 8-10 nm. E) EM 4800x showing sarcomere Z line disruption. F) EM 2900x of affected vessel.

to have IBM. Left biceps muscle biopsy revealed prominent perivascular deposition of amyloid leading to the diagnosis of amyloid myopathy. There was also rare rimmed vacuoles, fiber muscle necrosis and perivascular inflammation. There was HLA Class 1 sarcolemmal expression, CD3+ T cells with rare invasion of non-necrotic muscle fibers, and variable expression of phosphorylated neurofilaments on SMI-31 antibody staining (Fig. 2). Immunochemistry for transthyretin was weakly positive but negative for beta-

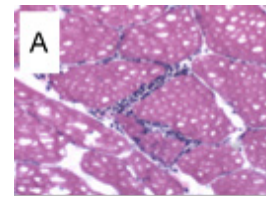

H\&E

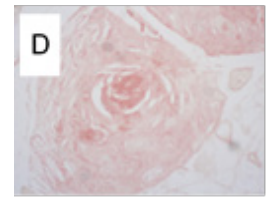

Congo Red

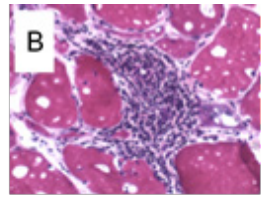

H\&E

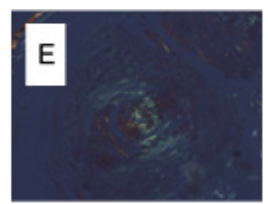

Congo Red (polarized)

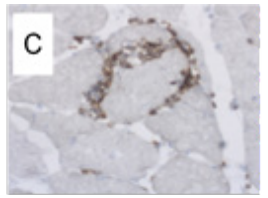

Immunohistochemistry (IHC)

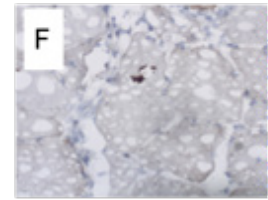

IHC - SMI-31 antibody
Figure 2. Biopsy of left biceps muscle. A) H\&E stain showing endomysial inflammation. B) H\&E stain showing perivascular inflammation. C) IHC showing predominantly CD3+ T-cells. D) Non-polarized Congo red stain showing amyloid deposits in blood vessel wall. E) Polarized Congo Red stain confirming the presence of amyloid in thickened blood vessel walls. F) IHC SMI-31 Ab showing phosphorylated neurofilament deposition. amyloid protein and kappa and lambda light chains. A search for systemic amyloidosis, including bone marrow and rectal biopsy, echocardiography and genetic testing for transthyretin mutations was unfruitful.

\section{Discussion}

IBM classically causes distal arm and proximal leg weakness also known as MP4 but can have a pleomorphic presentation (Table 1). The differential diagnosis of IBM includes inflammatory, autoimmune and genetic diseases, and in some cases, degenerative or even metabolic disorders. Table 1 lists confounders of IBM by phenotypic presentation.

Recognition and description of the clinical pattern of weakness remains an essential part of the diagnostic approach to myopathies. The patients described here, however, illustrate that tissue diagnosis is of key importance. While our patients had clinical presentations consistent with IBM as determined by neuromuscular experts, muscle histopathology revealed a different diagnosis with quite different management.

The clinical scenario was slightly different in our cases. Cases 1, 3 and 4 presented for evaluation of myopathy and were ultimately diagnosed with amyloidosis. Case 2 had an established diagnosis of systemic amyloidosis and only later amyloid myopathy. In cases 1, 2 and 3, the distribution of weakness was striking and notable for distal arm and proximal leg weakness. In case 4 , there was weakness more notable in the finger flexors when compared to finger extensors without lower extremity weakness. Therefore, all patients required muscle biopsy to disprove the initial erroneous diagnosis of IBM.

Our study has several limitations. The number of cases is small. The data was retrospectively abstracted. We did not measure antibody titers to cyclic nucleotidase-1A as patients were evaluated before the availability of these autoantibodies. Furthermore, these autoantibodies have been increasingly described to be non-specifically elevated in various inflammatory myopathies and autoimmune rheumatologic disorders. For example, these are found in Sjögren's syndrome (23-36\%), systemic lupus erythematosus (14-20\%) and dermatomyositis (15\%). . $^{14,15}$

For case 4, follow up duration was short. Though histopathologic evidence of inflammation was described previously in amyloid myopathy leading to an erroneous diagnosis of polymyositis, rare rimmed vacuoles would be quite an unusual finding. ${ }^{4}$ The rarity of these vacuoles renders them 
Table 1: IBM phenotypes and differential diagnosis according to clinical patterns.

\begin{tabular}{|c|c|}
\hline IBM Phenotypes ${ }^{1}$ & Differential diagnosis \\
\hline Distal Arm/Proximal Leg (MP4) & Myotonic MD², Sarcoidosis ${ }^{3}$, Amyloid Myopathy ${ }^{4}$ \\
\hline Distal Weakness (MP2) & Myotonic $\mathrm{MD}^{2}, \mathrm{ALS}^{5}$ \\
\hline Quadriceps Atrophy & Becker $\mathrm{MD}^{6}, \mathrm{LGMD}^{6}$, Emery-Dreifuss ${ }^{6}, \mathrm{PM}^{6}, \mathrm{SMA}^{6}$ \\
\hline Limb-girdle pattern (MP1) & $\begin{array}{l}\text { Polymyositis }{ }^{7}, \text { LGMD } \text { Pompe }^{8}, \text { Amyloid Myopathy }^{4} \text {, } \\
\text { Sarcoidosis }\end{array}$ \\
\hline Bulbar (MP7) - dysphagia & $\mathrm{MG}^{2}, \mathrm{LEMS}^{2}, \mathrm{ALS}^{2}, \mathrm{LGMD}^{2}$ \\
\hline
\end{tabular}

1. Dimachkie et al., 2014; ${ }^{6}$ 2. Barohn et al., 2014 $;{ }^{7}$ 3. Wolfe et al., 1987; ${ }^{9} 4$. Gert et al., 1996; ${ }^{3}$ 5. Barohn et al., 2013; ${ }^{10}$ 6. neuromuscular.west. edu/muscdist/quad.html; 7. Dimachkie et al., 2014;"11 8. Manganelli et al., 2013;12 9. Hinterbuchner et al., $1964 .{ }^{13}$

non-diagnostic. Furthermore, these have been non-specifically observed in other disorders such as polymyositis, hereditary IBM, and neurogenic atrophy. ${ }^{16}$

All of our cases fulfilled the 2011 ENMC clinical and laboratory criteria for IBM (Supplementary Table 1). ${ }^{17}$ Cases 3 and 4 even fulfilled criteria for clinically defined IBM and probable IBM respectively. This is given pathologic evidence of HLA class 1 expression in Case 3 and inflammation with myofiber invasion in Case 4. This highlights the importance of obtaining muscle biopsy in patients with IBM and that Congo Red staining should be part of the routine evaluation of IBM muscle. Beyond that, it raises questions about the specificity of the ENMC 2011 IBM criteria. For instance, if Congo Red stain was not done in cases 3 and

\begin{tabular}{|c|c|c|}
\hline $\begin{array}{c}\text { Clinical \& } \\
\text { Laboratory Features }\end{array}$ & Classification & Pathological Features \\
\hline $\begin{array}{l}\text { Duration }>12 \text { months } \\
\text { Age at onset }>45 \text { yrs } \\
\text { Quads weak } \geq \text { hip flex } \\
\text { and/or } \\
\text { FF weak }>\text { should abd } \\
\text { sCK not }>15 x \text { LN }\end{array}$ & $\begin{array}{c}\text { Clinico- } \\
\text { Pathologically } \\
\text { Defined IBM }\end{array}$ & $\begin{array}{l}\text { Endomysial inflammation \& } \\
\text { Rimmed vacuoles \& either } \\
\text { Protein accumulation } \\
\text { (amyloid or other proteins) } \\
\text { or } \\
15-18 \mathrm{~nm} \text { filaments }\end{array}$ \\
\hline $\begin{array}{l}\text { Same as in Clinico- } \\
\text { pathologically Defined } \\
\text { IBM except } \\
\text { Quads weak } \geq \text { hip flex } \\
\text { and } \\
\text { FF weak > should abd }\end{array}$ & $\begin{array}{l}\text { Clinically-Defined } \\
\text { IBM }\end{array}$ & $\begin{array}{l}\text { One or more of: } \\
\text { Endomysial inflammation } \\
\text { Rimmed vacuoles } \\
\uparrow \text { MHC1 } \\
\text { Protein accumulation* } \\
\text { (amyloid or other proteins) } \\
\text { 15-18nm filaments }\end{array}$ \\
\hline $\begin{array}{l}\text { Same as in Clinico- } \\
\text { pathologically Defined } \\
\text { IBM except } \\
\text { Quads weak } \geq \text { hip flex } \\
\text { or } \\
\text { FF weak > should abd }\end{array}$ & $\begin{array}{l}\text { Probable IBM } \\
\text { *amyloid = Congo-red, } \\
\text { other proteins = p62, S }\end{array}$ & $\begin{array}{l}\text { Same as clinically defined } \\
\text { IBM } \\
\text { crystal violet, or thioflavine T/S } \\
\text { SMI- } 31 \text {, or TDP-43 }\end{array}$ \\
\hline
\end{tabular}

Supplementary Table 1: IBM diagnostic criteria (adapted from The ENMC IBM research diagnostic criteria 2011 ${ }^{17}$ ).
4, the diagnosis would have been IBM based on these criteria (Supplementary Table 1).

\section{Conclusion}

Amyloid myopathy may clinically mimic IBM and manifest as an initial or delayed presentation of primary systemic amyloidosis. Despite the availability of antibody testing, muscle biopsy remains critically important in the evaluation of patients suspected to have IBM and serves to distinguish IBM from its many mimics.

\section{Corresponding author:}

Anai C. Hamasaki, MD

Email: $\underline{\text { Anai-Hamasaki@ouhsc.edu }}$

\section{Bibliography}

${ }^{1}$ Kyle, R.A. and M.A. Gertz, Systemic amyloidosis. Crit Rev Oncol Hematol, 1990. 10(1): p. 49-87.

${ }^{2}$ Kelly, J.J., Neurologic complications of primary systemic amyloidosis. Rev Neurol Dis, 2006. 3(4): p. 173-81.

${ }^{3}$ Gertz, M.A. and R.A. Kyle, Myopathy in primary systemic amyloidosis. J Neurol Neurosurg Psychiatry, 1996. 60(6): p. 655-60.

${ }^{4}$ Smestad, C., et al., Amyloid myopathy presenting with distal atrophic weakness. Muscle Nerve, 2004. 29(4): p. 605-9.

${ }_{5}^{5}$ Tuomaala, H., et al., Amyloid myopathy: a diagnostic challenge. Neurol Int, 2009. 1(1): p. e7.

${ }^{6}$ Dimachkie, M.M. and R.J. Barohn, Inclusion body myositis. Neurol Clin, 2014. 32(3): p. 629-46, vii. 
${ }^{7}$ Barohn, R.J., M.M. Dimachkie, and C.E. Jackson, A pattern recognition approach to patients with a suspected myopathy. Neurol Clin, 2014. 32(3): p. 569-93, vii.

${ }^{8}$ Abuzinadah, A.R., J.T. Joseph, and L. Korngut, Amyloid myoneuropathy mimicking inclusion body myositis. Can J Neurol Sci, 2013. 40(2): p. 255-8.

${ }^{9}$ Wolfe, S.M., et al., Myopathy in sarcoidosis: clinical and pathologic study of four cases and review of the literature. Semin Arthritis Rheum, 1987. 16(4): p. 300-6.

${ }^{10}$ Barohn, R.J. and A.A. Amato, Pattern-recognition approach to neuropathy and neuronopathy. Neurol Clin, 2013. 31(2): p. 343-61.

${ }^{11}$ Dimachkie, M.M., R.J. Barohn, and A.A. Amato, Idiopathic inflammatory myopathies. Neurol Clin, 2014. 32(3): p. 595-628, vii.

${ }^{12}$ Manganelli, F. and L. Ruggiero, Clinical features of Pompe disease. Acta Myol, 2013. 32(2): p. 82-4.

${ }^{13}$ Hinterbuchner, C.N. and L.P. Hinterbuchner, Myopathic Syndrome in Muscular Sarcoidosis. Brain, 1964. 87: p. 355-66.

${ }^{14}$ Lloyd, T.E., et al., Cytosolic 5'-Nucleotidase 1A As a Target of Circulating Autoantibodies in Autoimmune Diseases. Arthritis Care Res (Hoboken), 2016. 68(1): p. 66-71.

${ }^{15}$ Herbert, M.K., et al., Disease specificity of autoantibodies to cytosolic 5'-nucleotidase $1 \mathrm{~A}$ in sporadic inclusion body myositis versus known autoimmune diseases. Ann Rheum Dis, 2016. 75(4): p. 696-701.

${ }^{16}$ Dimachkie, M.M. and R.J. Barohn, Distal myopathies. Neurol Clin, 2014. 32(3): p. 817-42, x.

${ }^{17}$ Rose, M.R. and E.I.W. Group, 188th ENMC International Workshop: Inclusion Body Myositis, 2-4 December 2011, Naarden, The Netherlands. Neuromuscul Disord, 2013. 23(12): p. 1044-55. 